\title{
Manufacturing of Potato Chips and its Quality Improvement
}

\author{
Bhupesh Goyal ${ }^{1 *}$ and Pravin Goyal ${ }^{2}$ \\ ${ }^{1}$ Mechanical Engineering Department, Parul University, Vadodara, India
}

${ }^{2}$ Proprietor, Navin Gruh Udyog, Sayaji Wafers, Vadodara, India

\begin{abstract}
The current trend in the food industry has shown tremendous growth due to change in lifestyle as well as the variety of taste available outside. Potato chips are on the variety of food that people uses with breakfast as snacks. Chips are prepared by slicing potatoes and frying the chips in vessel contained oil at controlled temperature using the furnace. The crisp of chips depends on a number of factors including the quality of oil, the temperature of the oil, frying time, quality of potato and moisture contained in the chips. This review concludes with a case study of a manufacturing unit producing potato chips.
\end{abstract}

\section{Keywords: Frying pan; Slicer; Dryer; Potato peeler}

\section{Introduction}

The potatoes are used since ancient times for food. Today rare fast food is available in the market without potato in its different forms i.e. fries chips. The consumption of potato has been increased because of its high fiber content as well as sweet taste. At the same time, the potato chips are high in fat and calories which increases the risk of weight gain and obesity. Chips are low in vitamins and minerals. It contains sodium which is not good for heart functioning as it increases the blood pressure. The production of potato chips carried out from storing the potato at low temperature to final packaging.

\section{Manufacturing of Potato Chips}

\section{Raw material storage at low temperature}

To convert the raw potatoes into chips it has to pass from different processes. It has to withstand high temperature in furnace oil to make crispy chips. As the oil temperature is high $\left(195^{\circ} \mathrm{C}\right)$, the chips may get reddish color if it is not able to withstand high temperature. To avoid this difficulty the raw potatoes are stored in the low-temperature range which increases its heat taking capacity. The regular potatoes used in the kitchen are not required such property as it does not need such high temperature. Thus this is the first step of the chips manufacturing process which also improves its quality in output [1].

\section{Potato peeling}

The skin of potato must be removed as it is to be considered as waste as it contains dust, sand particles, and other impurities. To remove the skin, manual peeling can be done but to carry out on large scale it is quite difficult. To carry out the process on large scale, an automatic peeling machine is required. It contains silicon carbide coated plate which rotates on a shaft attached with the motor. Figure 1 shows the inside view of the peeling machine. The plate surface is wavy so that the potatoes at upper portion move downward during the rotation of the plate and the downward potatoes go upward. As the skin comes in contact with the silicon carbide plate it gets eroded and by continuous water supply the skin is removed and washed away through the hole provided in the corner. Using the centrifugal force of the rotor, the potatoes are removed from the peeling section by taking hinge upward direction. Now the potatoes are collected in a tank filled with water. The care has to be taken so that carbide particles from the peeling plate must not attach or get inside the potato [2].

\section{Cutting Chips using die}

The potatoes received after peeling washed again in the fresh water to remove the dust particles or other impurities. The potatoes are now inserted in the rotary die carrying cutting blades at the edges. As the rotor rotates, the potatoes are thrown away from the center due to the centrifugal force and then pass through the blades which cut the potatoes in round pieces chips. The shape of the chips depends on the blade shape. Simultaneously in more quantity potatoes are inserted in the die. The chips are now collected in the tank filled with water. The water level in the tank should be such that the chips can dip inside the water. To get better quality and appearance chips, the chips must remain inside water otherwise its surface get black color due to oxidation. Potassium aluminum sulfate is also known as Potassium Alum is used in the water to purify it and also provides whiteness to the chips. Figure 2 shows the 3rotary slicing machine.

\section{Dryer to remove water}

The wet chips from the tank are now filled in the dryer who is having perforated rotary plate rotated by the shaft power. Again due to centrifugal force, the chips are thrown away and strikes with the plate that water is removed. The complete dryness of the chips needs not to be achieved. Figure 3 shows the rotary dryer.

\section{Frying of chips}

The final stage of the manufacturing of the chips is the frying of chips in the pan filled with oil. Figure 4 shows the complete view of the setup. The setup consists of a frying pan of a rectangular shape with the size of $1800 \mathrm{~mm} \times 900 \mathrm{~mm} \times 300 \mathrm{~mm}$. The pan with the perforated plate is completely filled with vegetable oil through the sump available besides. As and when required the sump runs using pump maintains the level of oil in the pan for proper frying of chips. The frying temperature needs to be maintained at $195^{\circ} \mathrm{C}$. If temperature increases, it causes burning of chips at its outer boundary and also makes it reddish which changes

*Corresponding author: Bhupesh Goyal, Mechanical Engineering Department, Parul University, Vadodara, India, Tel: 02668260 312; E-mail: bhupesh.goyal@paruluniversity.ac.in

Received September 06, 2018; Accepted November 08, 2018; Published November 12, 2018

Citation: Goyal B, Goyal P (2018) Manufacturing of Potato Chips and its Quality Improvement. J Food Process Technol 9: 765. doi: 10.4172/2157-7110.1000765

Copyright: @ 2018 Goyal B, et al. This is an open-access article distributed under the terms of the Creative Commons Attribution License, which permits unrestricted use, distribution, and reproduction in any medium, provided the original author and source are credited. 
Citation: Goyal B, Goyal P (2018) Manufacturing of Potato Chips and its Quality Improvement. J Food Process Technol 9: 765. doi: 10.4172/21577110.1000765

the taste of the chips to bitter. As chips from the dryer entered in the frying pan, the oil comes upward as the chips contain water or moisture which causes bubble formation or boiling of water suddenly and water convert into steam. The salt is added as per taste in the frying pan itself

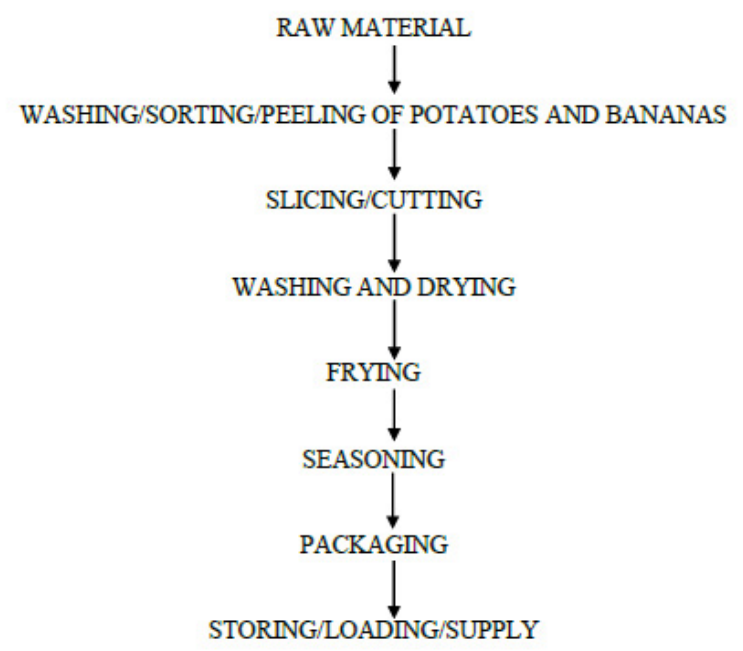

Figure 1: Technological process [1].
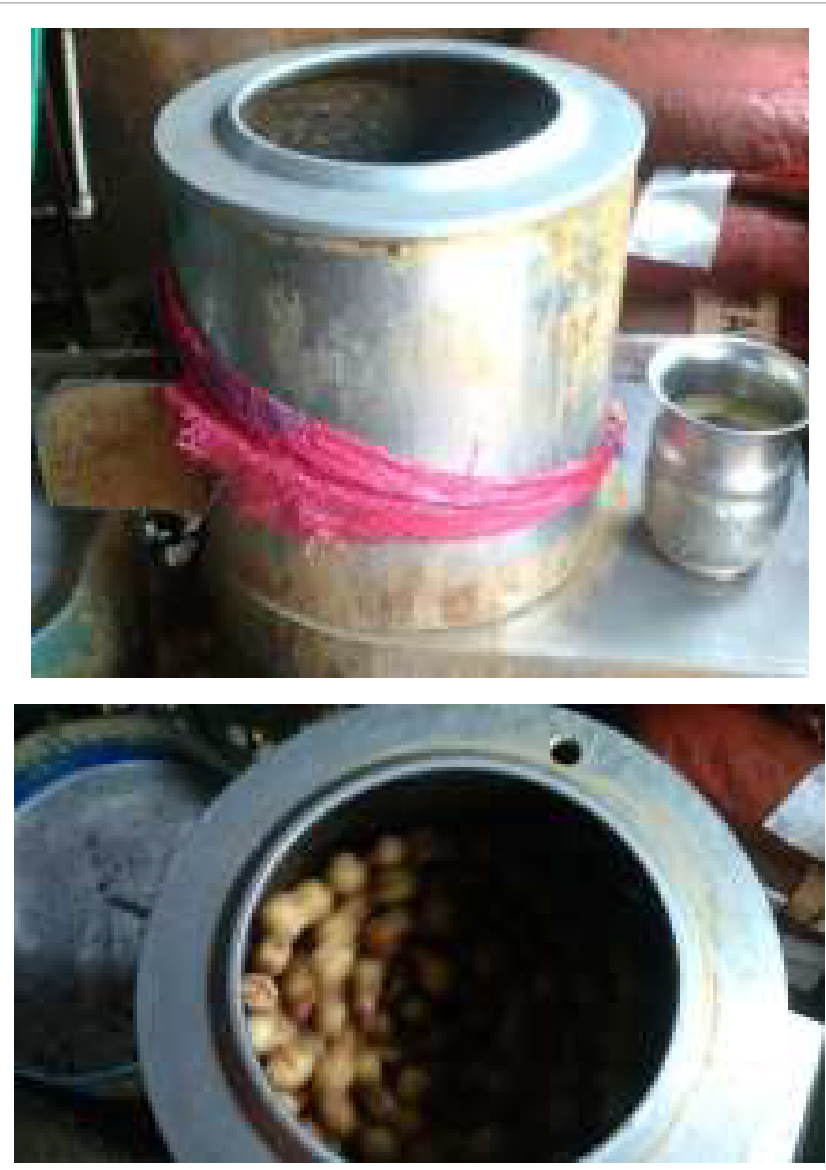

Figure 2: Peeling machine. to get a homogeneous taste of salt (Figure 5).

\section{Packaging}

Chips after frying received in a tray of rectangle size and distributed homogeneously so that each piece of chips will get proper cooling. After 3-4 minutes chips get cooled and it becomes crispy. After this packaging is carried by putting the chips in the plastic bags of required

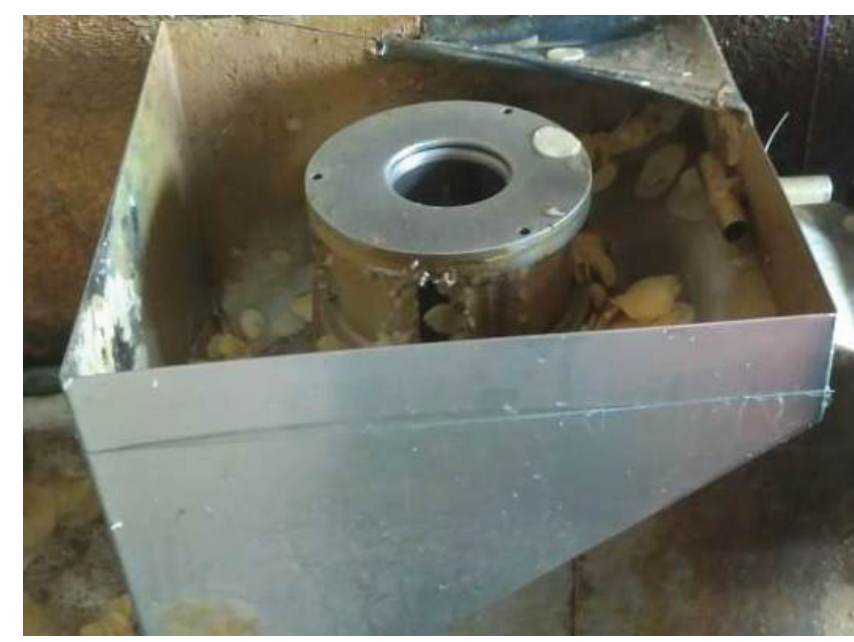

Figure 3: Potato chips slicing.

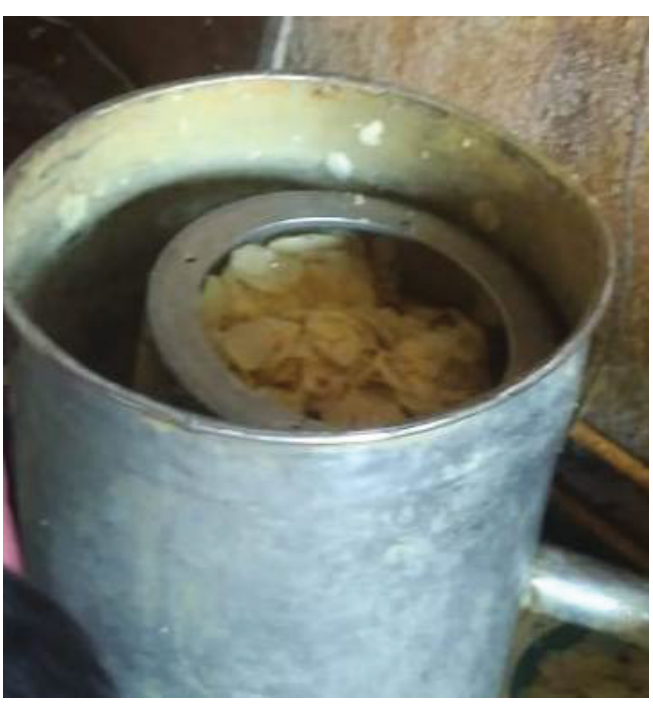

Figure 4: Rotary dryer.

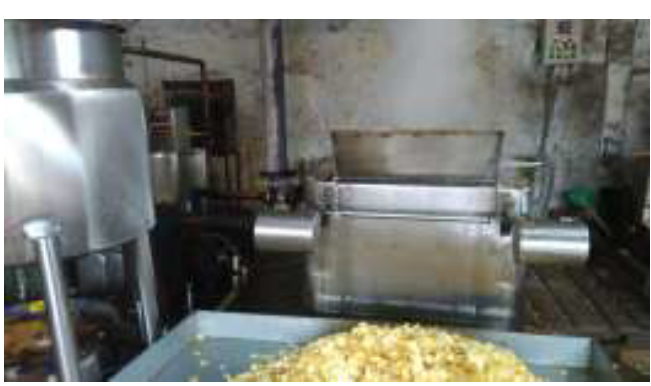

Figure 5: Setup of frying. 
Citation: Goyal B, Goyal P (2018) Manufacturing of Potato Chips and its Quality Improvement. J Food Process Technol 9: 765. doi: 10.4172/21577110.1000765

Page 3 of 3

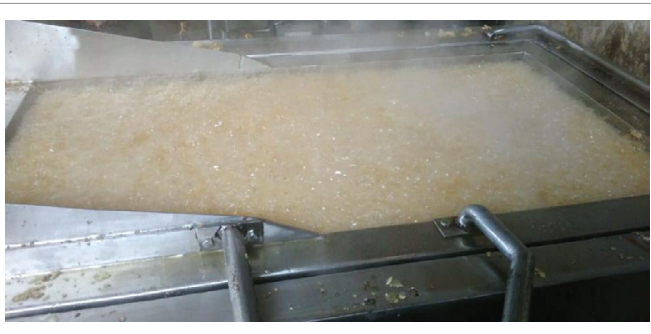

Figure 6: Frying.

size [3]. The packaging must be sealed airtight or using nitrogen which maintains the chips for a longer period of time (Figure 6).

\section{Conclusion}

The potatoes were not stored and the chips get a reddish color which made the taste of chips bitter.

The skin of potatoes was not removed properly which adhere to the outer boundary of the pieces of chips. During frying, this skin did not take higher temperature and get black or reddish color. Also, it ruined the physical appearance.

The carbide particles did not stick to the plate of peeling machine properly so it leaves plate surface and gets stuck into potato which finally ruptures the blades of the slicing machine. The rupture or wear of blades creates uneven pieces of chips during cutting.

Finally, the proper time must be given for the frying of the chips. Continuous stirring of the oil filled pan is required for the proper heating of individual chip homogeneously.

The temperature required is maintained $195^{\circ} \mathrm{C}$ for proper fomentation which also improves the quality of the chips.

\section{Acknowledgment}

Mr. Vishnubhai Agrawal and Mr. Pravin Goyal (Proprietor, Sayaji Wafers) are highly acknowledged for their support and suggestions.

\section{References}

1. North Eastern Development Finance Corporation Ltd (2017) Project Report on Potato and Banana Chips.

2. Wright RC, Davis ME, Hendel CE (1950) The Making of potato chips. Crops in Peace and War-The Yearbook of Agriculture.

3. Food and Agriculture Organization of the United Nations "Potato Crisps". 